ECCOMAS

Proceedia
COMPDYN 2021

$8^{\text {th }}$ ECCOMAS Thematic Conference on Computational Methods in Structural Dynamics and Earthquake Engineering M. Papadrakakis, M. Fragiadakis (eds.) Streamed from Athens, Greece, 28 - 30 June 2021

\title{
INVESTIGATIONS ON INTER-STOREY SEISMIC ISOLATION AS A TECHNIQUE FOR ADDING UPPER STOREYS
}

\author{
Enrico Bernardi ${ }^{12}$, Marco Donà ${ }^{2}$, Francesca da Porto ${ }^{1}$ and Ping Tan $^{2}$ \\ ${ }^{1}$ Department of Geosciences, University of Padova, Via Gradenigo 6, 35131 Padova, Italy \\ enrico.bernardi@dicea.unipd.it, francesca.daporto@unipd.it \\ ${ }^{2}$ Earthquake Engineering Research \& Test Center (EERTC), Guangzhou University, Guang Yuan \\ Zhong Rd. 248, 510405 Guangzhou, China \\ marco.dona.1@unipd.it, ptan@gzhu.edu.cn
}

\begin{abstract}
The inter-storey seismic isolation technique is becoming increasingly attractive for the seismic risk mitigation of buildings, also as alternative strategy to base isolation. The reasons for applying this technique can be various and of different nature, such as: architectural concerns, feasibility of construction, and performance benefits. An interesting application of this technique concerns its use for adding upper storeys to existing buildings, avoiding the increase of seismic forces on the substructure, or even reducing them. Therefore, this technique could also be effective for seismic retrofit applications, using the mass of the superstructure as a nonconventional TMD for controlling the dynamic response of the substructure.

Among the main issues concerning this application, there is the need to control the relative displacement between the two structural parts and the acceleration of the superstructure, while improving the seismic performance of the substructure.

In this paper, a multi-objective optimization method for the dynamic characteristics of the additional superstructure is presented, which uses a TMD approach and considers the performances of the substructure, isolation system and superstructure. A 3-storey (3 DOF) case study structure was taken as a reference, analysing a wide range of isolated masses, isolation periods and damping ratios. Time-history analyses are finally performed, based on the optimization results, in order to assess the effectiveness of both the optimization method and the isolation technique, also considering the structural non-linearity.
\end{abstract}

Keywords: Inter-Storey Isolation System (IIS), Tuned Mass Damper (TMD), MultiObjective Optimization, Frequency Response Functions, Time-History Analyses.

ISSN:2623-3347 @ 2021 The Authors. Published by Eccomas Proceedia.

Peer-review under responsibility of the organizing committee of COMPDYN 2021.

doi: 10.7712/120121.8709.18889 


\section{INTRODUCTION}

The inter-storey isolation system (IIS) is becoming increasingly attractive, as it allows greater freedom in the structural conception of skyscrapers and multi-purpose buildings by defining two independent structures, i.e. substructure and superstructure, which may have different forms, materials and uses (e.g. [1,2]). This represents both an advantage for architectural design and a sustainable solution for densely populated areas, as it allows significant savings on land use. Furthermore, in some cases, the base isolation technique encounters economic and technical issues that can prevent its application; for example, its application is often difficult and expensive in existing buildings, and generally more than applying isolation between storeys.

Moreover, the IIS technique can be applied to add extra storeys on the top of existing buildings, with appropriate vertical capacity, without increasing the base shear forces (e.g. [3, $4,5])$. In particular, the isolated superstructure, when properly designed, can be used as a Tuned Mass Damper (TMD) to improve the seismic behaviour of the substructure, thus making this technique very advantageous when retrofitting and elevation of the existing building are both required.

From a functional point of view, the IIS technique combines the functions of seismic isolation and mass damping; indeed, the isolation system is a filter for the inertial forces transmitted to the superstructure and, at the same time, induces the isolated superstructure to exert a mass damping action on the substructure, improving the seismic behaviour of the latter. In the literature, two main conceptual approaches can be identified, each of which focuses on one of the two main aspects of the IIS, i.e. seismic isolation or mass damping (TMD).

Studies that address the problem from the point of view of seismic isolation evaluate the interaction between the superstructure and the substructure, the effects of the higher vibration modes and their possible coupling, using frequency and mass ratios as design parameters (e.g. $[6,7,8,9,10,11,12])$, but also the optimal parameters of additional Fluid Viscous Dampers (FVDs) installed in the IIS (e.g. [2, 13, 14]). 3 degree-of-freedom (DOF) models (e.g. [8, 9]) or multi DOF models (e.g. $[2,10,12])$ are generally adopted, depending on the purpose of the study.

Other researchers, on the other hand, approach the study of the IIS following the strategy of mass damping, which is based on the principle of TMD, and considering the peculiarities of the IIS, namely the fact that the isolated mass also performs structural and housing functions in addition to that of dynamic control. The design parameters are the mass and frequency ratios between the TMD and the primary structure, and the damping ratio provided by the TMD. Generally, frequency and damping ratios are optimized for set values of mass ratio, using simplified 2 DOF models.

The concept of TMD for structural application was first introduced by [15], who applied a secondary mass to a single degree-of-freedom (SDOF) system harmonically excited. [16] first established a TMD design approach to suppress the peak displacement of an undamped SDOF system (primary structure) sinusoidally excited. This approach is based on the observation that all frequency response functions (FRFs) of the combined 2 DOF system (SDOF primary structure with TMD), obtained for various damping ratios of TMD, pass through two specific points ("fixed point theory"). Following the same design approach of [16], [17] derived the TMD design formula to optimizes the performance of the undamped SDOF primary structure in the case of harmonic excitation at the base (more useful for earthquake engineering applications). Later, [18] proposed the equations for the optimal tuning parameters of the TMD in the case of damped SDOF primary structures. Then, [19] first conceived the idea of using a heavily damped vibration absorber (or TMD) to reduce the seismic response of the structure. 
In particular, he suggested to tune the TMD in resonance with the primary structure and showed that its best performance is obtained when the two complex vibration modes of the resulting 2-DOF system have similar damping ratios, and approximately equal to the mean damping ratio between those of the TMD and the structure.

For several years, studies have focused on TMDs with low mass ratios, which are also often susceptible to tuning problems (e.g. [20]). Only more recently, various investigations have been directed towards TMDs with large mass ratios, also called nonconventional TMDs, given the growing interest in innovative applications such as mega-sub-control-structures (MSCS), inter-storey isolation systems and sliding roof systems (e.g. [21]). These TMDs have shown greater robustness than those with low mass ratios, due to their lower sensitivity to tuning (e.g. [22]). In particular, [23] showed that the design formulas proposed by [19] do not guarantee the equality of the modal damping ratios of the two complex modes in the case of TMD with mass ratios greater than 0.005 ; therefore, through a numerical procedure, [23] provided new design equations that result in equal damping ratios and equal frequencies for the two vibration modes. [24] validated the TMD tuning equations obtained by [23], proposing an analytical formulation that matches the same results. [25] proposed an alternative approach for tuning TMDs with large mass ratios, still equalizing the damping ratios but, at the same time, minimizing the maximum FRF amplitude associated with the displacement of the primary structure. The TMD damping ratio according to this last approach is significantly lower than that deriving from the model of [23], despite ensuring a similar efficiency of the TMD. [26] proposed an energy-based design methodology for non-conventional TMDs, which maximizes the ratio between the energy dissipated by the isolation system (located between the masses of a 2DOF system) and that of the input earthquake. [27] compared different optimization approaches, minimizing the variance of the responses (i) in displacement or (ii) in acceleration of the primary structure, or (iii) maximizing the energy dissipated by the TMD system (like [26]). [28] provided the equations for optimal TMD tuning, using a 2 DOF reducedorder model without damping in the main structure, minimizing (i) the maximum amplitudes of the base shear force under harmonic excitations, or (ii) the variance of the base shear force under a band-limited random excitation of white noise. [21], similarly to [25], proposed a TMD optimization approach that approximately equals the damping ratios of the 2 DOF system and, at the same time, minimizes its overall response; in particular, in addition to minimizing the FRF of the displacement of the primary structure, as done by [25], [21] simultaneously minimized the FRF of the acceleration of the nonconventional TMD, holding the latter also structural functions.

This paper investigates the inter-storey isolation system (IIS) as a technique for adding upper storeys to an existing building, improving at the same time its seismic behaviour. A 3storey case study structure (without considering the IIS) is taken as a reference, analysing a wide range of isolated masses, isolation periods and damping ratios. First, some assessments are carried out on the effects that the IIS parameters (normalized to the substructure characteristics) have on the dynamic response of the substructure, using the complex modal analysis and evaluating the FRFs of the equivalent 2 DOF system. An alternative approach for tuning the IIS (or TMD) parameters is then proposed, carrying out two multi-objective optimizations that consider the performance of the substructure along with that of the isolation system (to limit the P- $\triangle$ effects on the substructure, $O P T 1$ ) or along with that of the superstructure (to limit the TMD accelerations, OPT2). It is worth noting that high damping values in the IIS are effective in limiting the relative displacement of the isolators (and therefore the P- $\Delta$ effects) but could have negative effects on the overall structural behaviour, especially on the superstructure and its content (e.g. [2, 13, 14, 29]). Parametric time-history analyses are finally performed, based on the optimal solutions obtained for the IIS parameters, in order to assess the 
effectiveness of the optimization method and therefore of the isolation technique, also considering the structural non-linearity.

\section{CASE STUDY STRUCTURE AND PARAMETER DEFINITION}

For reasons of clarity, even if the study presented here is of general applicability, reference was made to a generic three-storey structure, representative of regular RC frame buildings with a fundamental period of about $0.3 \mathrm{~s}$. The building is shown in Figure 1 and is characterized by the following floor masses, $m_{1}=m_{2}=350 \mathrm{t}$ and $m_{3}=300 \mathrm{t}$, and by the following storey stiffnesses, $k_{l}=k_{2}=k_{3}=780 \mathrm{kN} / \mathrm{mm}$. Assuming that the structure can support additional floors (by its own capacity or through appropriate interventions), it is intended to add an isolated superstructure above it (which can therefore be defined as substructure), whose total mass is given by the masses of the isolation layer and the additional floors.

Conceptually, the IIS subdivides the structure into three main parts, i.e. the substructure, the isolation system and the superstructure. As said before, some relevant studies focusing on the aspect of seismic isolation analyse the structure using 3 DOF or multi DOF models, while other studies adopting TMD approaches evaluate the structural response using 2 DOF reduced-order models. However, as demonstrated in various studies (e.g. [7, 12]), when the mass ratio $(\mu)$ between the isolated mass and the mass of the substructure is less than 1 , there is a "mass damping" behaviour, and therefore it is possible to neglect the higher vibration modes of the superstructure, simplifying the system with an equivalent 2 DOF model.

Thus, considering values of $\mu$ in this study up to 1 , both substructure and isolated superstructure were modelled with an equivalent SDOF model. In particular, the substructure is characterized by an angular frequency $\left(\omega_{S D O F}\right)$ of $21 \mathrm{rad} / \mathrm{s}$ (i.e., vibration period of about $0.3 \mathrm{~s}$ ) and a damping ratio $\left(\xi_{S D O F}\right)$ of $5 \%$; its equivalent mass $\left(m_{S D O F}\right)$ is $606 \mathrm{t}$ and corresponds to the modal mass of the first vibration mode of the 3 DOF structure, calculated as in Equation 1, where $\phi_{1}$ is the first eigenvector normalized to 1 and $\mathbf{M}_{\mathbf{s t}}$ the mass matrix of the structure. On the other hand, the isolated superstructure is characterized by the parameters $m_{I S}$ (sum of the masses of isolation and superstructure), $\omega_{I S}$ and $\xi_{I S}$ (angular frequency and damping ratio of the isolation system), as shown in Figure 1; the relation between $m_{I S}$ and $\omega_{I S}$ is given in Equation 2, where $k_{I S}$ is the IIS stiffness. Considering the parameters of the isolated superstructure normalized to those of the substructure, as generally done in TMD approaches, the mass $(\mu)$ and frequency $(v)$ ratios in Equation 3 can be defined, which together with $\xi_{I S}$ represent the dimensionless parameters of the nonconventional TMD to be optimized. Lastly, Equation 4 shows the matrices of mass $(\mathbf{M})$, damping $(\mathbf{C})$ and stiffness $(\mathbf{K})$ of the 2 DOF reduced-order model (Figure $1 \mathrm{~b}$ ), as a function of the aforementioned parameters.

In this study, the optimization is performed for the parameters $v$ and $\xi_{I S}$, considering the range from 0.05 to 1 for $v$ and the range from 0.01 to 2 for $\xi_{I S}$, for set values of $\mu(0.25,0.5$, 0.75 and 1$)$.

$$
\begin{gathered}
m_{S D O F}=\phi_{1}^{T} \mathbf{M}_{\mathbf{s t}} \phi_{1} \\
\omega_{I S}=\sqrt{\frac{k_{I S}}{m_{I S}}} \\
\mu=\frac{m_{I S}}{m_{S D O F}} ; v=\frac{\omega_{I S}}{\omega_{S D O F}} \\
\mathbf{M}=\left[\begin{array}{ll}
1 & 0 \\
0 & \mu
\end{array}\right] ; \mathbf{C}=\left[\begin{array}{cc}
2 \xi_{S D O F} \omega_{S D O F}+2 \mu \xi_{I S} v \omega_{S D O F} & -2 \mu \xi_{I S} v \omega_{S D O F} \\
-2 \mu \xi_{I S} v \omega_{S D O F} & 2 \mu \xi_{I S} v \omega_{S D O F}
\end{array}\right] ; \mathbf{K}=\left[\begin{array}{cc}
\omega_{S D O F}^{2}+\mu v^{2} \omega_{S D O F}^{2} & -\mu v^{2} \omega_{S D O F}{ }^{2} \\
-\mu v^{2} \omega_{S D O F}{ }^{2} & \mu v^{2} \omega_{S D O F}^{2}
\end{array}\right]
\end{gathered}
$$




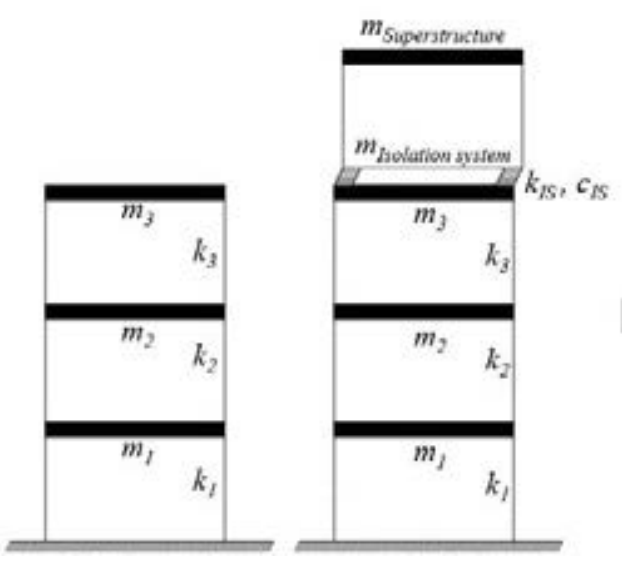

(a)

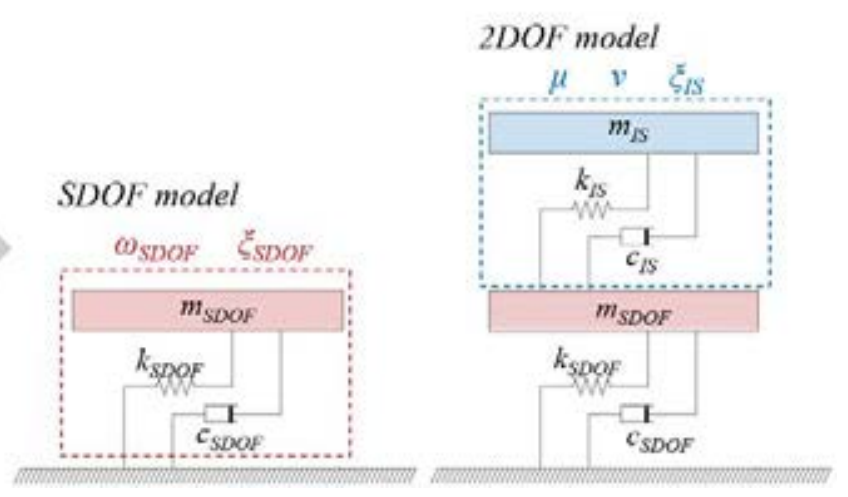

(b)

Figure 1: (a) MDOF models of the structure without and with IIS. (b) Related reduced-order models.

\section{ASSESSMENT OF STRUCTURAL BEHAVIOUR IN FREQUENCY-DOMAIN}

\subsection{Complex modal analysis}

In traditional structural design, damping is assumed proportional to mass and stiffness and the results from classical modal analysis are not affected by it (as the $\mathbf{C}$ matrix is not included in the problem formulation). When using non-proportional damping (e.g., in the case of IIS, where the damping is concentrated in some parts of the structure), the actual modal characteristics are different from the classical ones, and require a non-classical or complex modal analysis for their evaluation (e.g. [30]).

In this study the state-space formulation is adopted, which allows to decouple the equations of motion transforming the system with $n$ second-order differential equations into a system with $2 n$ first-order differential equations, where $n$ is the DOF number, namely:

$$
\dot{\mathbf{v}}(t)=\mathbf{A} \mathbf{v}(t)+\mathbf{I}_{\mathbf{g}} \ddot{u}_{g}(t)
$$

where $\mathbf{v}(t)$ is the state space vector at time $t, \mathbf{A}$ is the state space matrix, $\mathbf{I}_{\mathbf{g}}$ is the input vector and $\ddot{u}_{g}(t)$ is the applied input process. $\mathbf{v}(t), \mathbf{A}$ and $\mathbf{I} \mathbf{g}$ are defined below:

$$
\mathbf{v}(t)=\left[\begin{array}{l}
\mathbf{x} \\
\dot{\mathbf{x}}
\end{array}\right] ; \quad \mathbf{A}=\left[\begin{array}{cc}
\mathbf{0} & \mathbf{I} \\
-\mathbf{M}^{-1} \mathbf{K} & -\mathbf{M}^{-1} \mathbf{C}
\end{array}\right] ; \quad \mathbf{I}_{\mathbf{g}}=\left[\begin{array}{c}
\mathbf{0} \\
-\boldsymbol{\tau}
\end{array}\right]
$$

where $\mathbf{x}$ and $\dot{\mathbf{x}}$ are the relative displacement and relative velocity vectors, respectively; $\mathbf{I}$ is the unitary matrix of dimensions $2 \times 2 ; \mathbf{M}, \mathbf{K}$ and $\mathbf{C}$ are the system matrices defined in Equation $4 ; \tau$ is the unitary vector of dimensions $2 \times 1$. Therefore, the eigenvalue problem can be defined as:

$$
\mathrm{A} \boldsymbol{\psi}_{i}=\lambda_{i} \psi_{i}
$$

where $\lambda_{i}$ represents the eigenvalues and $\psi_{i}$ the eigenvectors.

Since the main objective of this study is the control of the seismic response of the substructure through the IIS, Figure 2 shows the results relating to the second vibration mode of the 2 DOF model (i.e., the mode associated with the deformation of the substructure), for $\mu=0.5$, values of $v$ equal to $0.2,0.4$ and 0.6 , and values of $\xi_{I S}$ in the range $0-2$. 

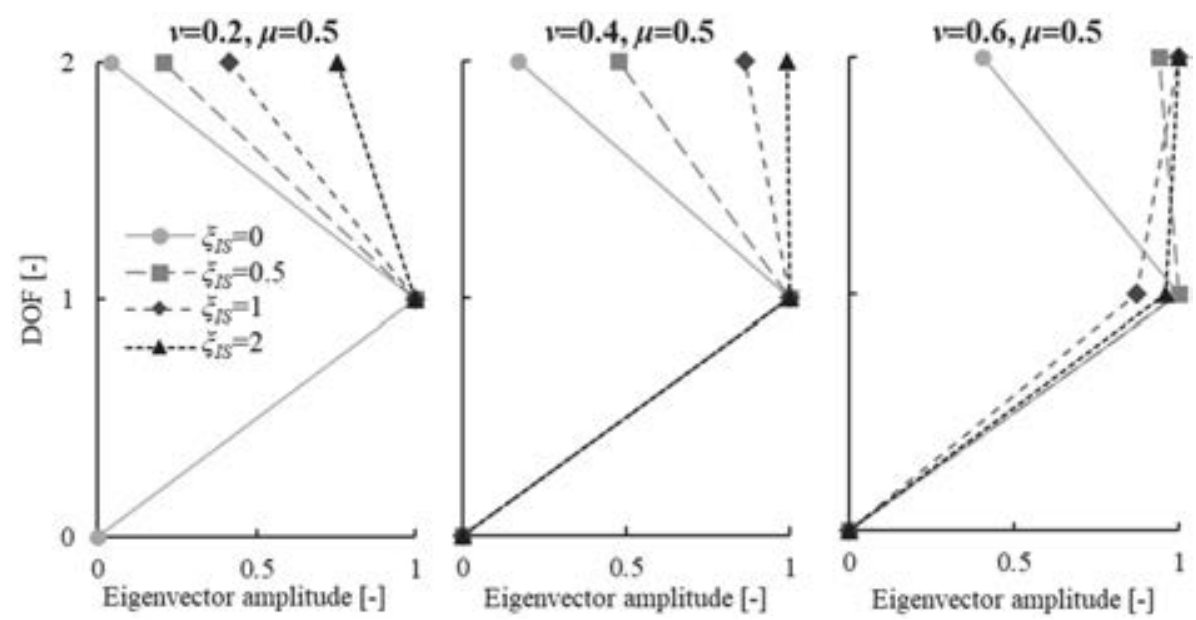

(a)
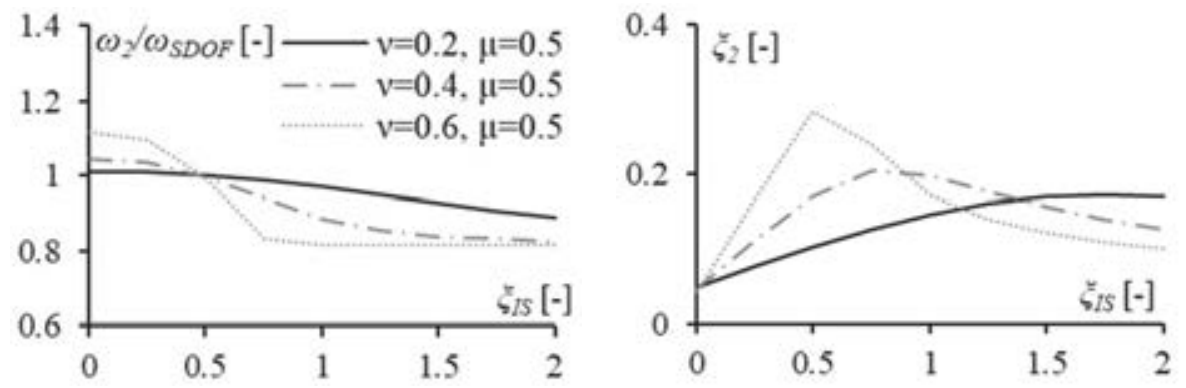

(b)

Figure 2: Complex modal analysis results of 2 DOF system, for $\mu=0.5, \nu=0.2,0.4,0.6, \xi_{I S}=0$ - 2: (a) eigenvectors of the second mode; (b) normalized frequency $\omega_{2}$ and damping ratio $\xi_{2}$ of the second mode, as a function of $\xi_{I S}$.

Figure $2 \mathrm{a}$ shows that, for low to medium values of the frequency $(v)$ and damping $\left(\xi_{I S}\right)$ ratios, the masses move in a decoupled way (characteristic behaviour of the isolated structures, due to the filter action exerted by the IIS), while increasing the value of the previous parameters the two masses reduce their relative displacement until, beyond certain values of $v$ and $\xi_{I S}$, they move together. Instead, Figure $2 \mathrm{~b}$ shows that, as $\xi_{I S}$ increases, the frequency of the second mode $\left(\omega_{2}\right)$ is reduced, while the damping ratio of the second mode $\left(\xi_{2}\right)$ first increases and subsequently, for high $\xi_{I S}$ values, it decreases. In particular, the peak of $\xi_{2}$ seems to be corresponding to the change in concavity of the $\omega_{2} / \omega_{\text {SDOF }}$ curve. These variations are greater for higher $v$ values (i.e., for more rigid IIS), as the resulting 2 DOF system is more coupled in terms of mass displacement. In fact, a "perfect coupling" (for sufficiently high $v$ and $\xi_{I S}$ values) will lead to a single vibration mode having a modal mass equal to $\left(m_{I S}+m_{S D O F}\right)$ and a modal damping ratio equal to that of the substructure, $\xi_{S D O F}$ (which justifies the reduction of $\xi_{2}$ for high $\xi_{I S}$ values).

\subsection{Frequency Response Functions}

To analyse the influence of the parameters $\mu, v$ and $\xi_{I S}$ on the actual structural response, the frequency response functions (FRFs) of the equivalent 2 DOF systems (obtained parametrically by varying the values of $\mu, v$ and $\xi_{I S}$ indicated in Section 2) were evaluated. FRFs express how a sinusoidal signal with a given input frequency is transferred within the system. In particular, the FRFs associated with the displacements $(\mathbf{H}(\omega))$ and accelerations $\left(\mathbf{H}_{a}(\omega)\right)$ of the various DOF of the system can be defined as follow, where $\omega$ is the excitation vibration frequency: 


$$
\begin{aligned}
& \mathbf{H}(\omega)=-\left(-\omega^{2} \mathbf{M}+i \omega \mathbf{C}+\mathbf{K}\right)^{-1} \mathbf{M} \tau \\
& \mathbf{H}_{a}(\omega)=-\omega^{2} \mathbf{H}(\omega)+\tau
\end{aligned}
$$

Specifically, the investigated FRFs concern the drift of the substructure (i.e., of the lower mass) $\left|\mathbf{H}_{1}(\omega)\right|$, the drift of isolation (i.e., between the two masses) $\left|\mathbf{H}_{2}(\omega)-\mathbf{H}_{1}(\omega)\right|$, and the acceleration of the superstructure (i.e., of the upper mass) $\left|\mathbf{H}_{a 2}(\omega)\right|$. Figure 3 shows the trend of the selected FRFs with respect to the variation of $\omega$ and one of the main parameters $\left(\mu, v, \xi_{I S)}\right.$ at a time - therefore as 3D surfaces - assuming $\mu=0.5, v=0.4$ and $\xi_{I S}=0.5$ as reference values for the other parameters. The following observations can be drawn:

- Increasing the values of $\mu$ results in a reduction of $\left|\mathbf{H}_{1}(\omega)\right|$ and $\left|\mathbf{H}_{a 2}(\omega)\right|$, and therefore in a better structural performance in terms of drift of the substructure and acceleration of the superstructure; instead, the influence of $\mu$ on $\left|\mathbf{H}_{2}(\omega)-\mathbf{H}_{1}(\omega)\right|$ (i.e., on the isolation drift) is not very significant.

- Low values of $v$ allow to limit the acceleration of the superstructure; intermediate values of $v$ (e.g., between 0.4 and 0.6) seem the most effective solution to reduce the drift of the substructure; on the other hand, high values of $v$ are optimal for reducing the isolation drift, even if they induce significant amplifications both on the substructure drift and on the superstructure acceleration.

- The increase in the $\xi_{I S}$ values provides an effective initial reduction of the substructure drift and of the superstructure acceleration, at least up to medium-high values of $\xi_{I S}$ (about 0.5 ), beyond which a further increase leads to a significant amplification of both $\left|\mathbf{H}_{1}(\omega)\right|$ and $\left|\mathbf{H}_{a 2}(\omega)\right|$. Instead, $\xi_{I S}$ is always effective in reducing the isolation drift, as also confirmed by other studies (e.g., $[2,13,14])$.
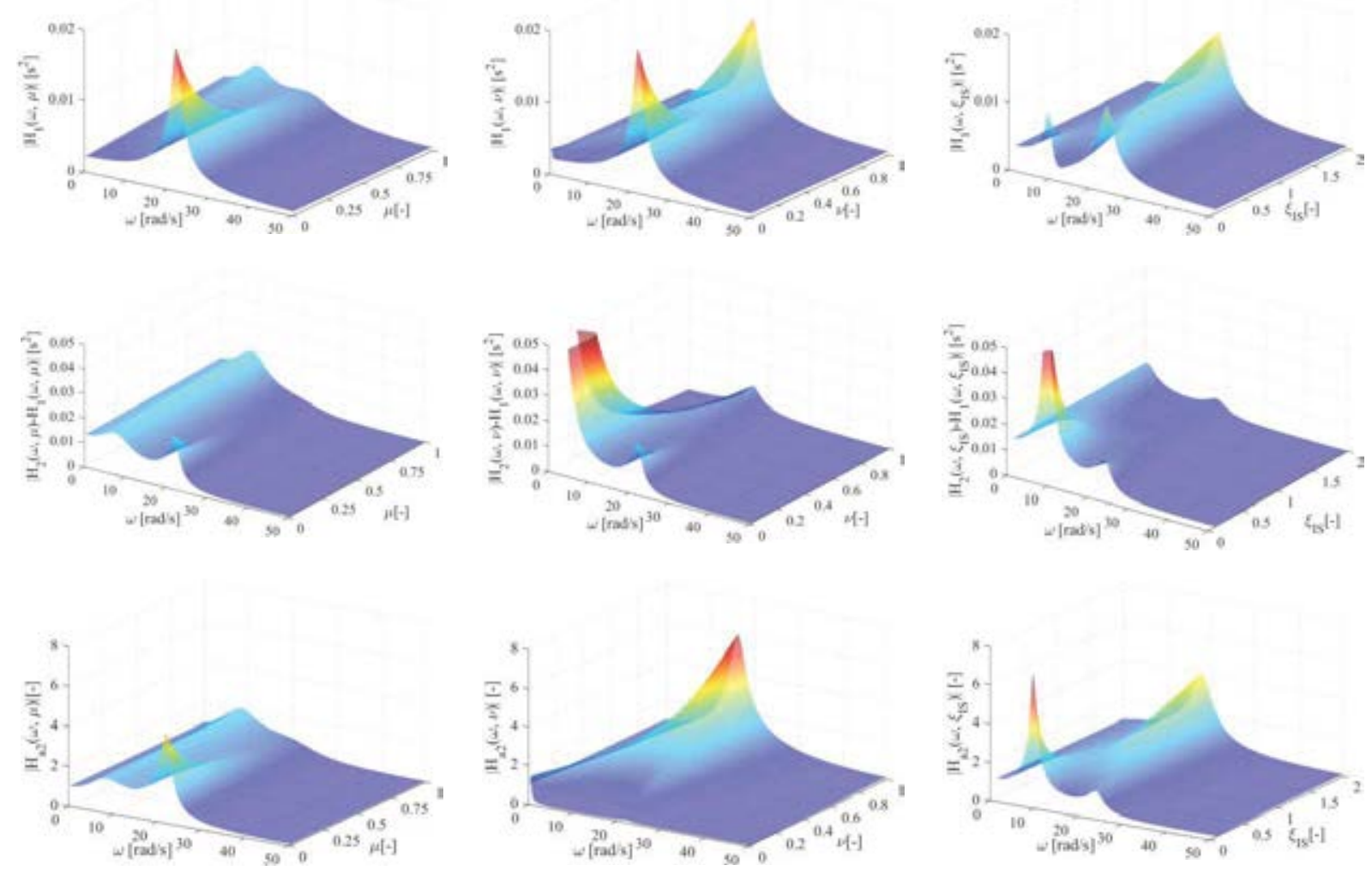

Figure 3: Trends of the FRFs as a function of $\mu, v$ and $\xi_{I S}$; reference parameters: $\mu=0.5, v=0.4$ and $\xi_{I S}=0.5$. 


\section{MULTI-OBJECTIVE OPTIMISATION}

\subsection{Proposed approach}

As seen in the Introduction, many of the literature studies on the optimization of TMD parameters (at least up to the most recent ones) seek the maximum effect of "mass damping" focusing on the minimization of the response of the primary system, thus neglecting the performance of the tuned mass (i.e., IIS drift and acceleration) in the optimization procedure. However, controlling the deflection of the isolators (and thus also the P- $\Delta$ effects on the substructure) and the acceleration of the superstructure can be of significant interest considering that the mass to be tuned is in fact a habitable building.

In order to model the stochastic nature of the seismic input, the Power Spectral Density (PSD) function of a zero-mean Gaussian stochastic process, $\mathbf{S}(\omega)$, is generally assumed; considering this type of process, the random vibration theory produces the following covariance matrices:

$$
\begin{aligned}
& \operatorname{Cov}\left[\mathbf{x x}^{T}\right]=\int_{-\infty}^{\infty} \mathbf{H}(\omega) \mathbf{S}(\omega) \mathbf{H}(\omega)^{* T} d \omega \\
& \operatorname{Cov}\left[\ddot{\mathbf{x}} \ddot{\mathbf{x}}^{T}\right]=\int_{-\infty}^{\infty} \mathbf{H}_{a}(\omega) \mathbf{S}(\omega) \mathbf{H}_{a}(\omega)^{* T} d \omega
\end{aligned}
$$

Furthermore, assuming the seismic excitation also as a white noise process, its PSD function is no longer dependent on $\omega\left(\mathbf{S}(\omega)=S_{0}\right)$; therefore, in order to obtain the optimal TMD parameters (i.e., $\mu, v, \xi_{I S}$, variables of $|\mathbf{H}(\omega)|$ and $\left|\mathbf{H}_{a}(\omega)\right|$ ), the minimization of the integrals in Equation 9 is equivalent to the minimization of the integrals in Equation 10, where $\boldsymbol{\sigma}^{2}$ and $\boldsymbol{\sigma}_{\mathbf{a}}{ }^{2}$ are the displacement and acceleration variance, respectively.

$$
\begin{aligned}
& \sigma^{2} / S_{0}=\int_{-\infty}^{\infty}|\mathbf{H}(\omega)|^{2} d \omega \\
& \sigma_{\mathrm{a}}^{2} / S_{0}=\int_{-\infty}^{\infty}\left|\mathbf{H}_{a}(\omega)\right|^{2} d \omega
\end{aligned}
$$

By defining the FRFs of the reference SDOF system, in terms of displacement $\left(\mathrm{H}_{S D O F}(\omega)\right)$ and acceleration $\left(\mathrm{H}_{\mathrm{a}, \mathrm{SDOF}}(\omega)\right)$ as:

$$
\begin{aligned}
& \mathrm{H}_{S D O F}(\omega)=-\left(-\omega^{2}+i \omega 2 \xi_{S D O F} \omega_{S D O F}+\omega_{S D O F}{ }^{2}\right)^{-1} \\
& \mathrm{H}_{a, S D O F}(\omega)=-\omega^{2} \mathrm{H}_{S D O F}(\omega)+1
\end{aligned}
$$

the following Performance Indices (PIs) can be defined:

$$
P I_{1}=\frac{\int_{-\infty}^{\infty}\left|\mathbf{H}_{1}(\omega)\right|^{2} d \omega}{\int_{-\infty}^{\infty}\left|\mathrm{H}_{S D O F}(\omega)\right|^{2} d \omega} \quad P I_{2}=\frac{\int_{-\infty}^{\infty}\left|\mathbf{H}_{2}(\omega)\right|^{2} d \omega-\int_{-\infty}^{\infty}\left|\mathbf{H}_{1}(\omega)\right|^{2} d \omega}{\int_{-\infty}^{\infty}\left|\mathrm{H}_{S O D F}(\omega)\right|^{2} d \omega} \quad P I_{3}=\frac{\int_{-\infty}^{\infty}\left|\mathbf{H}_{a, 2}(\omega)\right|^{2} d \omega}{\int_{-\infty}^{\infty}\left|H_{a, S D O F}(\omega)\right|^{2} d \omega}
$$

where, $P I_{1}$ and $P I_{2}$ are respectively the substructure drift variance and the isolation drift variance both normalized to the drift variance of the equivalent SDOF system, whereas $\mathrm{PI}_{3}$ is the 
superstructure acceleration variance normalized to the acceleration variance of the SDOF system. Therefore, based on these PIs, the objective functions (OFs) are defined as follow:

$$
O F_{1}=\min \left(P I_{1}\left(\xi_{I S}, v\right)\right) \quad O F_{2}=\min \left(P I_{2}\left(\xi_{I S}, v\right)\right) \quad O F_{3}=\min \left(P I_{3}\left(\xi_{I S}, v\right)\right)
$$

In particular, two multi-objective optimizations (OPT1 and $O P T 2)$ are performed, simultaneously minimizing $O F_{1}$ and $O F_{2}(O P T 1)$, or $O F_{1}$ and $O F_{3}(O P T 2)$, with the aim of controlling the substructure performance but, at the same time, also the deflection of the isolators or the acceleration of the superstructure.

In order to solve these multi-objective optimization problems, the well-known Nondominated Sorting Genetic Algorithm NSGA-II (e.g. [31, 32]) was used. This algorithm, widely used in practical optimization problems, first creates an initial parent population $\mathrm{P}_{0}$, of size $\mathrm{N}$, with a random process; then, sorts this population according to the non-domination criterion (i.e., assigning to each solution a rank based on its non-domination level - e.g. 1 for the best level, 2 for the next one and so on - and minimising it); subsequently, it generates an offspring population $\mathrm{Q}_{0}$, of size $\mathrm{N}$, through binary tournament selection, recombination and mutation operations. Once the initial parent $\left(\mathrm{P}_{0}\right)$ and offspring $\left(\mathrm{Q}_{0}\right)$ populations are defined, an iterative procedure is carried out for a set number of generations (to be defined according to the stability and convergence of solution). This procedure allows to determine the parent and offspring populations at generation $(k+1)$ starting from those at generation $(k)$. In particular, the solution $P_{k+1}$, of size $N$, is generated starting from the combined population $R_{k}=P_{k}+Q_{k}$, of size $2 \mathrm{~N}$, based on two cascading criteria: the solutions preferred are (i) those of lesser (nondomain) rank and, among solutions of equal rank, (ii) those located in a less crowded region (selected by a crowding comparison operator). Using the new parent population $\left(\mathrm{P}_{\mathrm{k}+1}\right)$, through a binary tournament selection, crossover and mutation operations, the new offspring population $\left(\mathrm{Q}_{\mathrm{k}+1}\right)$ is then created. This procedure continues until the last generation required. The main parameters of this algorithm are therefore the number of generations, the size of the population sought and the mutation and crossover probabilities; the values set in this study for these parameters are shown in Table 1.

\begin{tabular}{ll}
\hline Parameter & Value \\
\hline Generations & 120 \\
Population & 80 \\
Mutation probability & 0.1 \\
Crossover probability & 0.9 \\
\hline
\end{tabular}

Table 1: Parameters set for the NSGA-II algorithm.

\subsection{Optimization results}

The results obtained by the NSGA-II algorithm, i.e. the Pareto fronts, are shown in Figure 4 for both optimizations (OPT1 and OPT2). The main considerations are reported below.

- Overall, for the various cases analysed, the minimum values of $O F_{1}\left(O F_{1 m i n}\right)$ are approximately equal to 0.4 (both for $O P T 1$ and $O P T 2$ ), indicating a clear reduction of the substructure drift due to the application of the IIS (i.e., of the isolated superstructure).

- Then, while the minimization of $O F_{2}$ (in $O P T 1$ ) causes values of $O F_{1}$ greater than 1 , the minimization of $\mathrm{OF}_{3}$ (in $\mathrm{OPT2}$ ) allows values of $O F_{1}$ always less than 1; this means that the solutions aimed at minimizing the superstructure acceleration do not cause an increase in the substructure drift compared to the case without IIS (i.e., the SDOF system), as instead occurs when the minimization of the isolation drift is sought. 
- The values of $\mathrm{OF}_{3}$ show that the superstructure accelerations, when optimized, are significantly reduced compared to those of the existing substructure (i.e., the SDOF system).

- It is also worth noting that, as $\mu$ increases, the minimum values of $O F_{1}$ and $O F_{2}$ increase - for $O P T 1$ (Figure 4a), whereas the minimum values of $O F_{1}$ and $O F_{3}$ decrease - for OPT2 (Figure 4b).

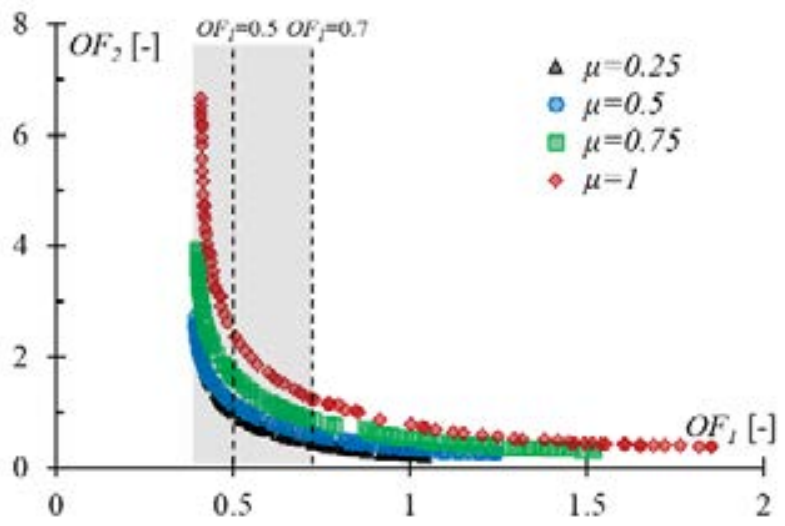

(a)

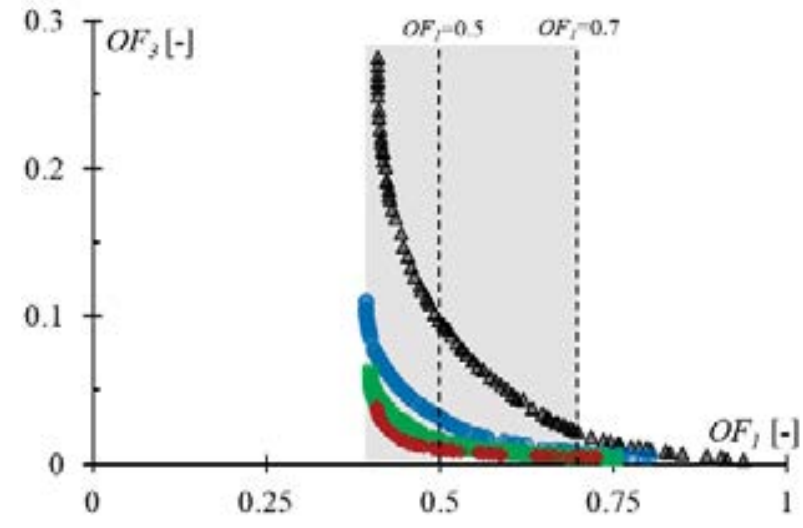

(b)

Figure 4: Pareto fronts (minimum values of OFs) for set values of $\mu$ : (a) OPT1; (b) OPT2.

Since $O F_{1}$ indicates the reduction of the variance of the substructure drift, a range from $O F_{1 \min }$ to $O F_{1}=0.7$ (i.e., $30 \%$ reduction) - as shown in Figure $4-$ is considered effective for the purposes of this study; in particular, three values of $O F_{1}$ were selected for subsequent evaluations (for both optimizations), namely $O F_{1 \min }, O F_{1}=0.5$ and $O F_{1}=0.7$.

Figure 5 shows the optimal values of the IIS (or TMD) parameters, for both OPT1 (Figure $5 \mathrm{a}$ ) and $O P T 2$ (Figure $5 \mathrm{~b}$ ), associated with the selected $O F_{1}$ values. In particular, the optimal values of $\xi_{I S}$ and $v$ are shown as a function of $\mu$, as well as the values of $O F_{2}$ for $O P T 1$ and the values of $\mathrm{OF}_{3}$ for $O P T 2$. The following observations can be derived:

- The $O F_{1 \min }$ solutions (which minimize the variance of the substructure drift) are the same for both $O P T 1$ and $O P T 2$ optimizations, as expected. For these solutions, as $\mu$ increases, the values of $\xi_{I S}$ increase and those of $v$ decrease; therefore, these optimal solutions range from the "mass damping" behaviour towards that of "intermediate seismic isolation", as already observed in previous studies.

- As regards the $O P T 1$ optimization, the reduction of $O F_{2}$ (i.e., of the variance of the isolation drift) is associated with the increase of the values of $\xi_{I S}$ and $v$, that is, with solutions that induce a greater coupling between the two structural parts.

- On the other hand, regarding the $O P T 2$ optimization, reductions of $O F_{3}$ (i.e., of the variance of the superstructure acceleration) are possible by increasing the values of $\xi_{I S}$ and reducing those of $v$, even significantly (especially for $O F_{l}=0.7$ and low $\mu$ values).

- Looking at the values of OFs as a function of $\mu$, it can be seen that $\mathrm{OF}_{2}$ is more sensitive for high values of $\mu$, whereas $O F_{3}$ for low values of $\mu$. In these respective $\mu$ ranges, multiobjective optimization is more effective and beneficial than single substructure performance optimization.

- The minimization of $O F_{3}$ (see the case $O F_{1}=0.7$ ), in the case of low values of $\mu$, is obtained by considerably reducing $v$ (i.e., increasing the filter action offered by the IIS) and significantly increasing $\xi_{I S}$ (beyond the critical damping value for the presented study). 

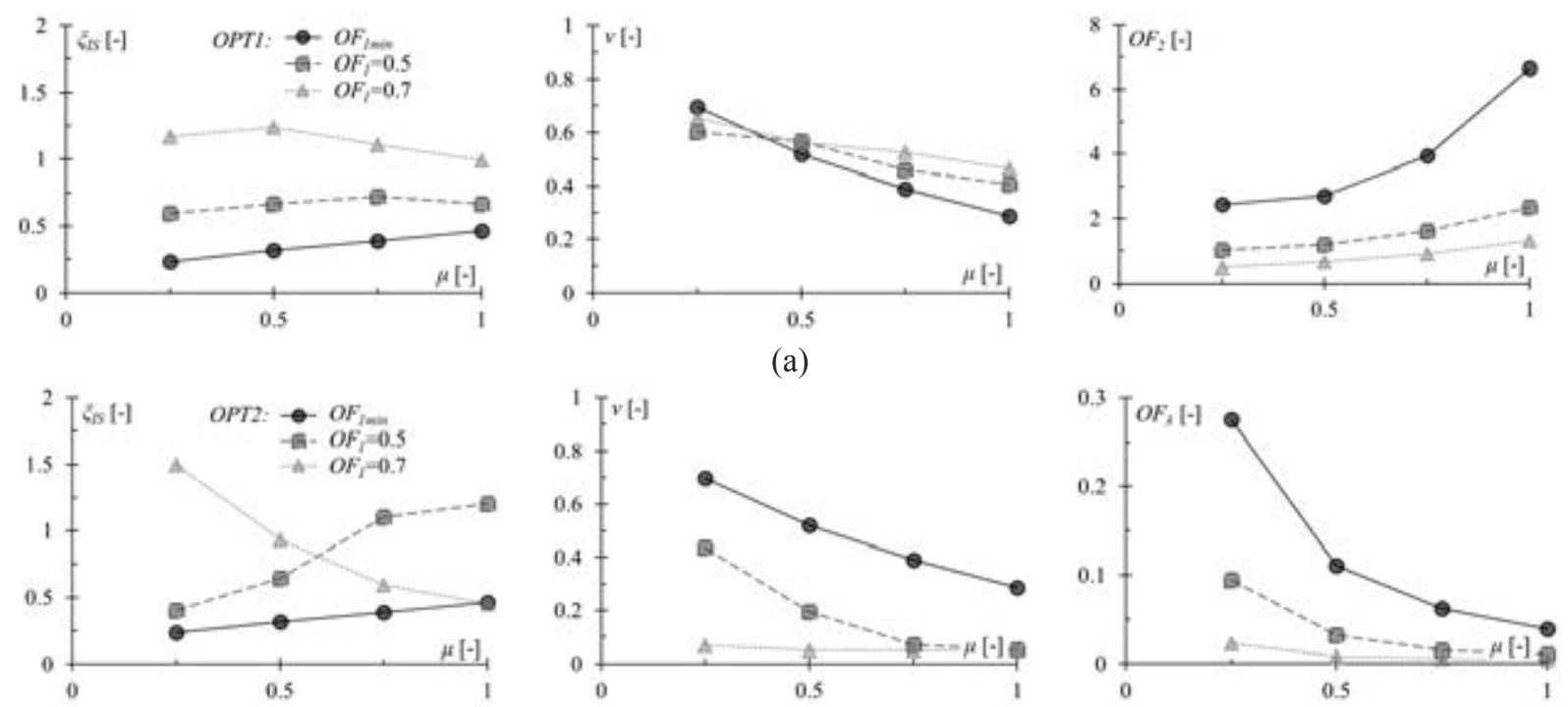

(b)

Figure 5: $O P T 1$ (a) and $O P T 2$ (b): optimal values of $\xi_{I S}, v, O F_{2}$ and $O F_{3}$ as a function of $\mu\left(O F_{l}=O F_{1 m i n}, 0.5,0.7\right)$.

\subsection{Comparison with other literature solutions}

The optimal values of the IIS parameters $\left(\xi_{I S}, v\right)$ obtained for the $O F_{1 \min }$ solution (equal for $O P T 1$ and OPT2), which minimize the displacement variance of the primary system, are compared in Figure 6 with other relevant literature solutions, as the mass ratio $\mu$ varies. These solutions are taken from: [17], who provided a closed-form solution for the TMD parameters in the case of an undamped primary SDOF system with base harmonic excitation; [25], who optimized the parameters of TMDs with large mass ratios by matching the damping ratios of the 2 DOF system while minimizing the maximum FRF amplitude associated with the displacement of the primary structure; [27], who proposed different approaches, including the minimization of the displacement variance of the primary structure (displacement approach) and the maximization of the energy dissipated by the TMD system (energy approach).

It is worth considering that the various solutions compared in Figure 6 refer to different values of the damping ratio of the primary structure $\left(\xi_{S D O F}\right)$, i.e.: $0 \%$ for $[17], 5 \%$ for $[25]$ and this study, and $2 \%$ for [27]. However, as discussed in previous study (e.g. [25, 27, 21]), reasonable variations of $\xi_{S D O F}$ do not significantly affect the optimal TMD parameters.

As can be seen from these comparisons, the results obtained for $O F_{1 \min }$ are very similar to those obtained by the other literature approaches, and in particular by [27] (displacement approach).
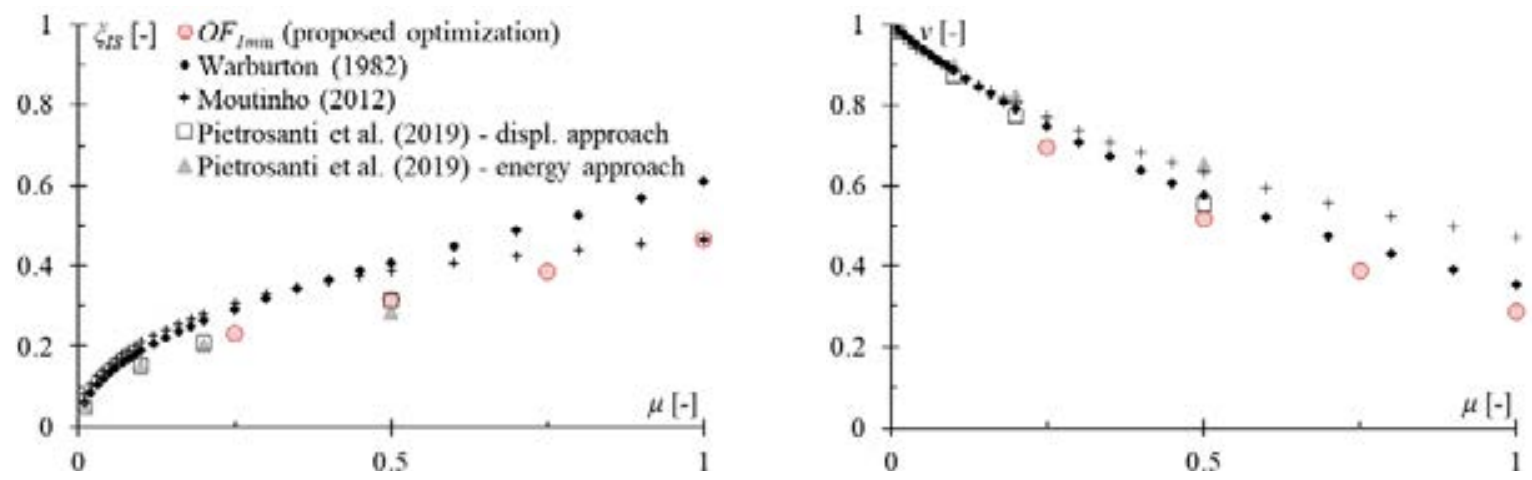

Figure 6: Optimal IIS parameters obtained in this study compared to other TMD solutions from the literature. 


\section{ASSESSMENTS THROUGH TH ANALYSES}

\subsection{Structure modelling and seismic input}

This section shows the results of a Time History (TH) analysis, carried out on the 3 DOF case study structure (see Section 2) without and with IIS, in order to evaluate the optimization solutions previously obtained and therefore the effectiveness of the IIS technique.

The structure (see Figure 7) was modelled as a lumped mass system with a non-linear structural behaviour, assuming the bi-linear hysteretic law of Takeda for the substructure, which is often used to describe the behaviour of ductile RC frames. This hysteretic model was calibrated on the elastic stiffness of each storey, assuming a ratio $r$ between the post-yield stiffness and the elastic stiffness equal to 0.2 , and an inter-storey drift $\delta_{y}$ at yield equal to $0.5 \%$. The superstructure was modelled as a linear SDOF system, adopting for the IIS parameters $\left(v\right.$ and $\left.\xi_{I S}\right)$ the optimal values obtained, associated with the mass ratio $\mu=0.5$ and the solutions of $O F_{1}=O F_{1 m i n}, 0.5$ and 0.7 , of both the optimizations performed (OPT1 and OPT2).
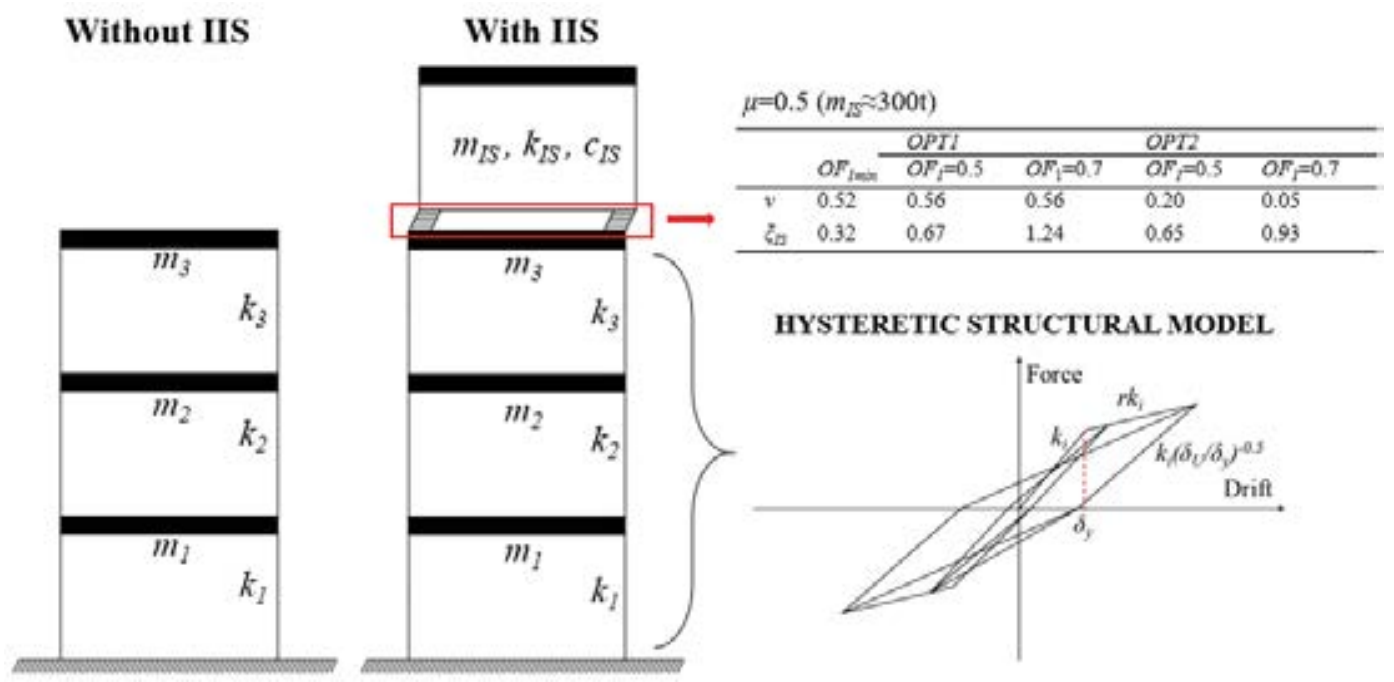

Figure 7: Hysteretic model of the substructure and optimal parameters of the IIS used for the TH analyses.

Time History analyses were performed for eight natural accelerograms, chosen among those of the European Strong-Motion Database (ESD, [33]). They were scaled to be compatible, on average, with the following elastic response spectrum of EC8: Type 1, $a_{g}=0.25 g$ (bedrock acceleration) and soil B (i.e., soil factor $S=1.2$ ); the peak ground acceleration PGA $\left(=a_{g}: S\right)$ is $0.3 g$. The main details of the natural records chosen are reported in Table 2, and the acceleration spectra are shown in Figure 8, together with that of EC8.

\begin{tabular}{llllllll}
\hline Ref. & Earthquake & Location & Date & $\mathrm{M}_{\mathrm{w}}$ & $\begin{array}{l}\text { Distance from } \\
\text { epicentre }[\mathrm{km}]\end{array}$ & $\begin{array}{l}\text { Fault } \\
\text { mechanism }\end{array}$ & $\begin{array}{l}\text { Scale } \\
\text { factor }\end{array}$ \\
\hline Acc.1 & Montenegro (aftershock) & Montenegro & $1979 / 05 / 24$ & 6.2 & 20 & thrust & 5.42 \\
Acc.2 & Campano Lucano & Italy & $1980 / 11 / 23$ & 6.9 & 33 & normal (y) & 3.02 \\
Acc.3 & Erzincan & Turkey & $1992 / 03 / 13$ & 6.6 & 13 & strike slip & 0.59 \\
Acc.4 & Ano Liosia & Greece & $1999 / 09 / 07$ & 6.0 & 18 & normal & 3.51 \\
Acc.5 & Campano Lucano & Italy & $1980 / 11 / 23$ & 6.9 & 33 & normal (x) & 3.04 \\
Acc.6 & Tabas & Iran & $1978 / 09 / 16$ & 7.3 & 57 & oblique & 1.71 \\
Acc.7 & Ano Liosia & Greece & $1999 / 09 / 07$ & 6.0 & 20 & normal & 0.32 \\
Acc.8 & Montenegro & Montenegro & $1979 / 04 / 15$ & 6.9 & 25 & thrust & 0.66 \\
\hline
\end{tabular}

Table 2: Information on the assumed accelerograms. 


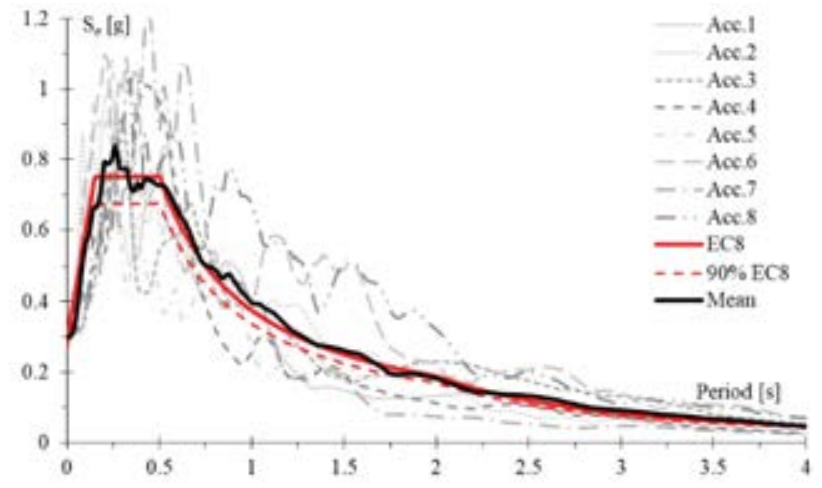

Figure 8: Acceleration response spectra compatible with the EC8 (Type 1) spectrum.

\subsection{Analysis results}

The main results obtained are shown in Figures 9 to 11. Figure 9 (for $O P T 1$ ) and Figure 10 (for $O P T 2$ ) shows the profiles of inter-storey drift and floor acceleration; in particular, they show the maximum values obtained from the analysis of the single accelerograms and the related average values. On the other hand, Figure 11 shows (for both OPT1 and OPT2) the results obtained for the isolation drift $\left(D_{I S}\right)$. The following considerations can be drawn:

- All the optimal solutions adopted made it possible to significantly reduce both the maximum inter-storey drifts and the maximum floor accelerations in the substructure, compared to the case without IIS. In particular, in the case without IIS, the maximum drift values exceeded the yield limit drift $\left(\delta_{y}=0.5 \%\right)$, causing inelastic structural deformations; instead, the optimal solutions of the IIS always allowed to remain in the elastic range.

- The $O F_{1 \min }$ solution, common to $O P T 1$ and $O P T 2$, is associated with the better performance of the substructure in terms of inter-storey drift, as expected.

- For $O P T 1$ optimization, as the accepted value of $O F_{1}$ increases (i.e., solutions $O F_{1}=0.5$ and then $O F_{1}=0.7$ ), the drift profiles of the substructure remain similar to those in the case of $O F_{1 m i n}$, i.e. there is no clear reduction in the substructure performance in terms of drifts, while it improves in terms of floor accelerations; then, as expected, the accelerations of the superstructure increase (albeit slightly), to the advantage of a greater reduction of the isolation drift (see Figure 11), due to the minimization of $O F_{2}$ along with $O F_{1}$.

- For $O P T 2$ optimization, as the accepted value of $O F_{1}$ increases (i.e., $O F_{1}=0.5$ and then $\left.O F_{1}=0.7\right)$, the response of the substructure worsens compared to the case of $O F_{1 \min }$, both in terms of maximum inter-storey drifts and floor accelerations; then, as expected, the accelerations of the superstructure are considerably reduced, due to the minimization of $\mathrm{OF}_{3}$ along with $O F_{1}$, at the expense of an increase in the deflection of the isolation system (with however acceptable values, as shown in Figure 11).

- It can be noted that moving away from the $O F_{1 \min }$ solution (e.g., $O F_{1}=0.7$ ), the $O P T 1$ results tend to give a more uniform response of the substructure along the building height, also reducing its variability with respect to the earthquake, whereas the OPT2 results provide a greater decoupling between the two structural parts (typical of seismic isolation), reducing the control function on the substructure. 

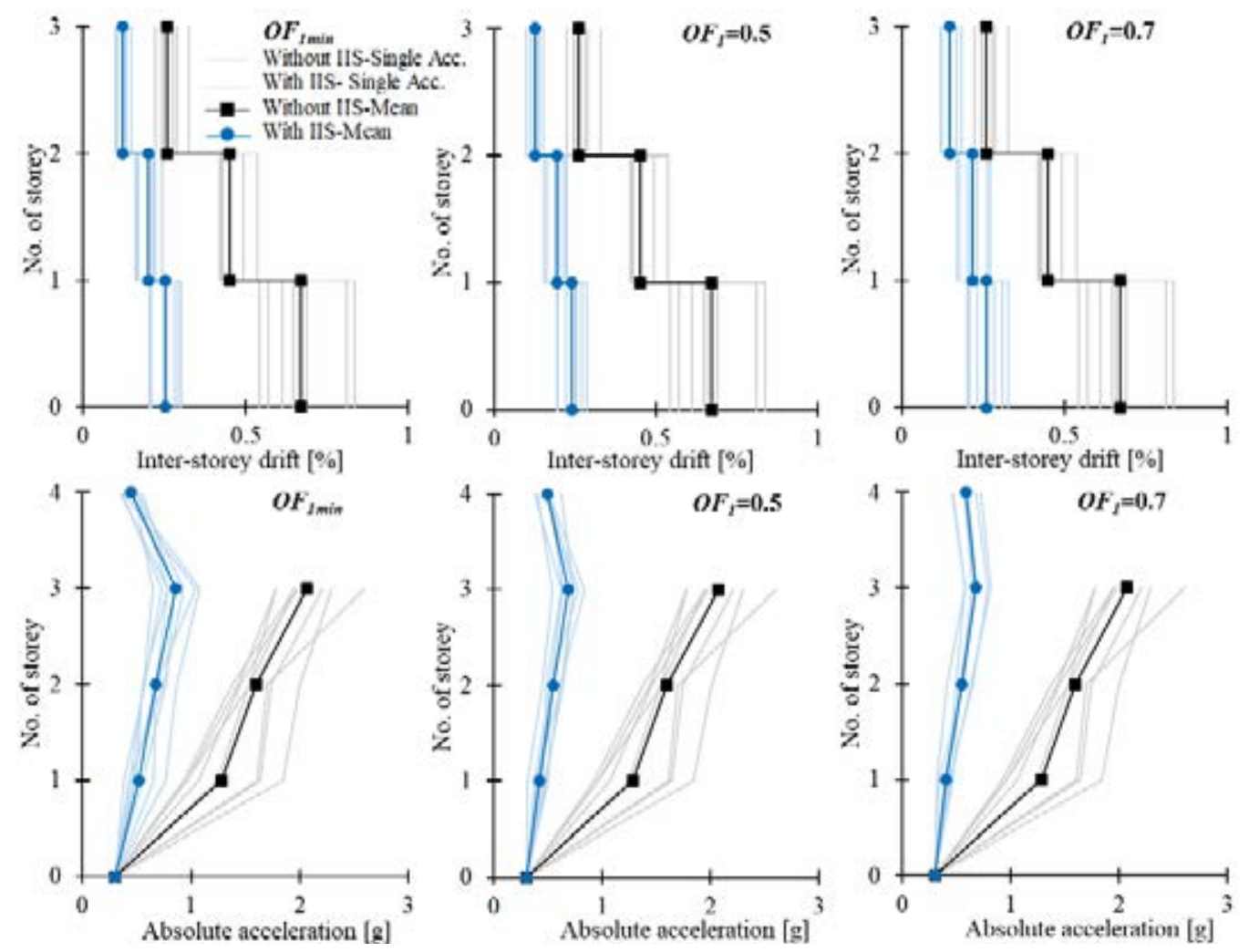

Figure 9: Profiles of inter-storey drift and floor acceleration, for OPT1 solutions.
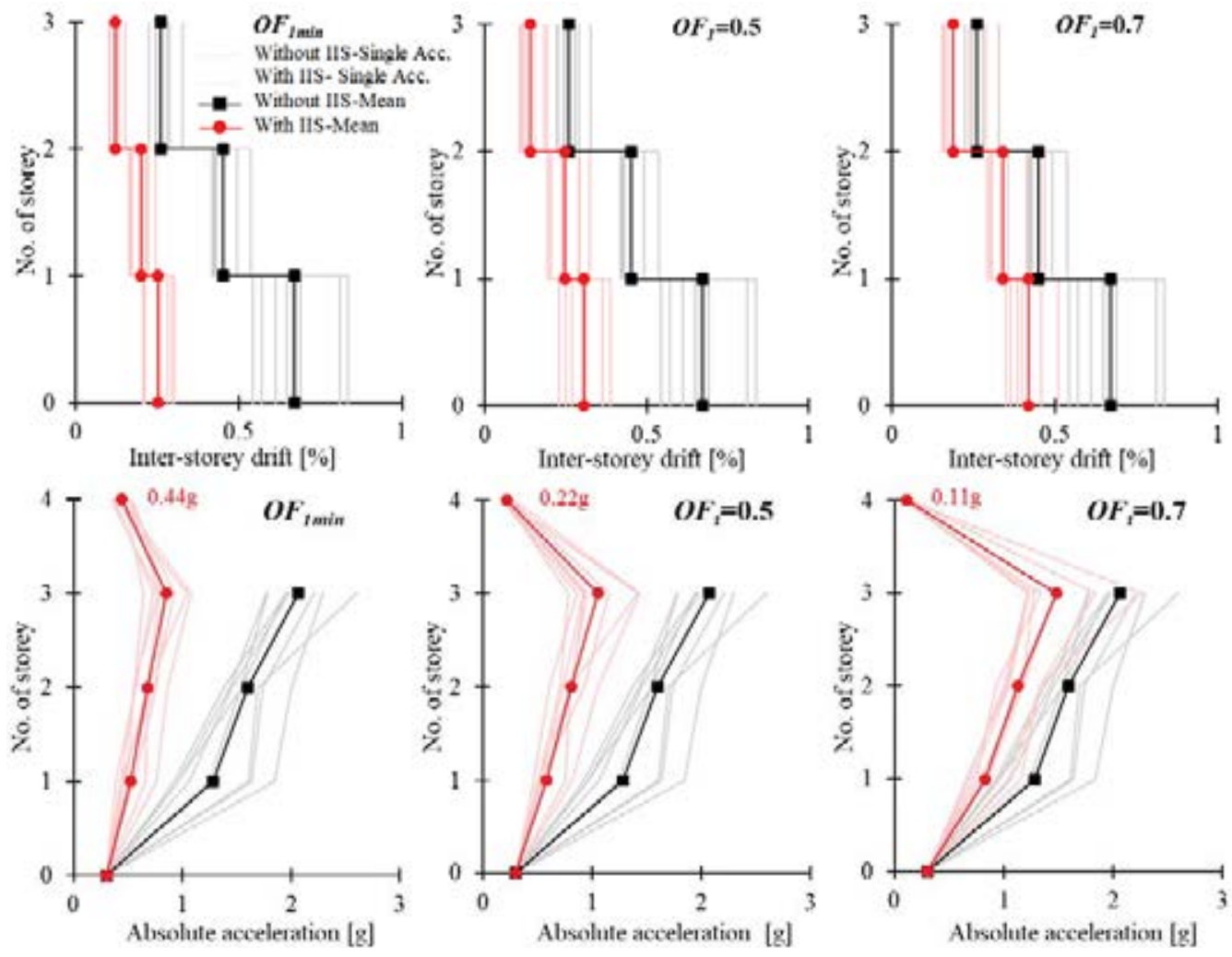

Figure 10: Profiles of inter-storey drift and floor acceleration, for OPT2 solutions. 

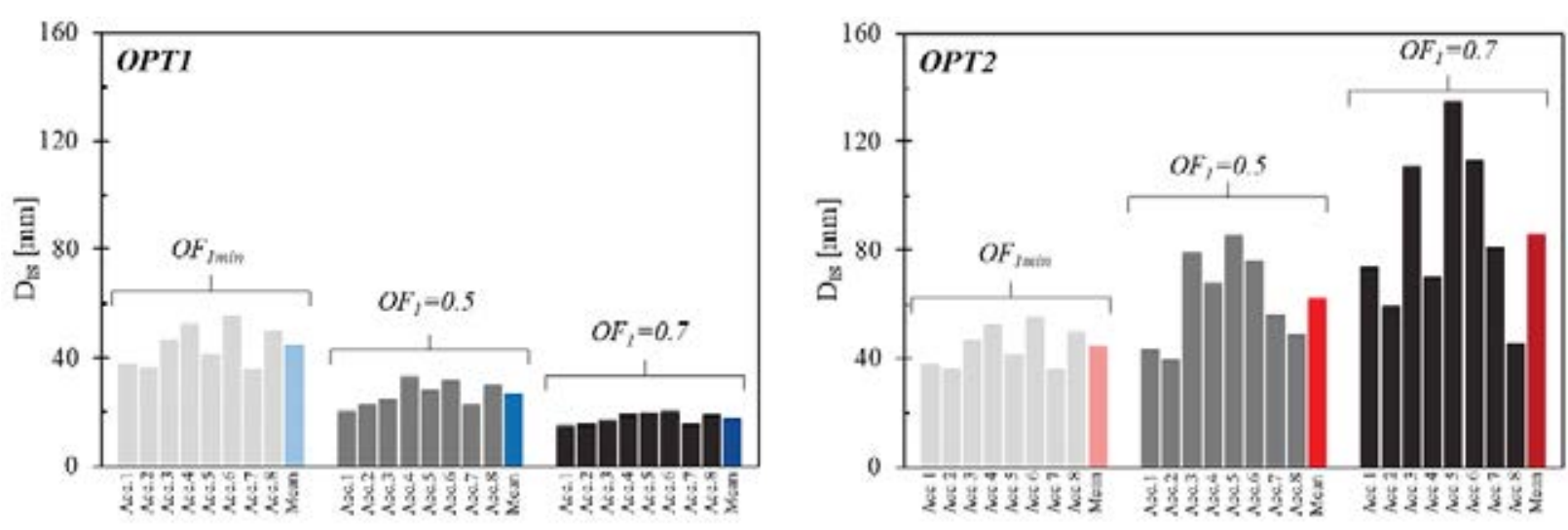

Figure 11: Isolation drift $\left(D_{I S}\right)$ values resulting from both optimizations (OPT1 and OPT2).

Figure 12 summarizes the main results obtained from the two optimizations. In particular, it shows the ratio between the average structural responses obtained for $O F_{1}=0.5$ and $O F_{1}=0.7$ and the respective ones obtained for $O F_{1 \min }$, considering the following performance parameters: substructure drift $\left(\theta_{\max }\right.$, associated with $\left.O F_{1}\right)$, isolation drift $\left(D_{I S}\right.$, associated with $\left.O F_{2}\right)$ and superstructure acceleration $\left(A_{U}\right.$, associated with $\left.O F_{3}\right)$.

Consistently with what was previously observed, and with respect to the $O F_{1 \min }$ solution (which minimizes the substructure drift), the OPT1 optimization allows to strongly reduce $D_{I S}$ (even more than 50\%) while maintaining good performances in terms of $\theta_{\max }$ and $A_{U}$, which are only slightly amplified. On the other hand, the OPT2 optimization allows strong reductions in $A_{U}$ (greater than 50\%) at the expense of appreciable amplifications of $\theta_{\max }$ and $D_{I S}$.
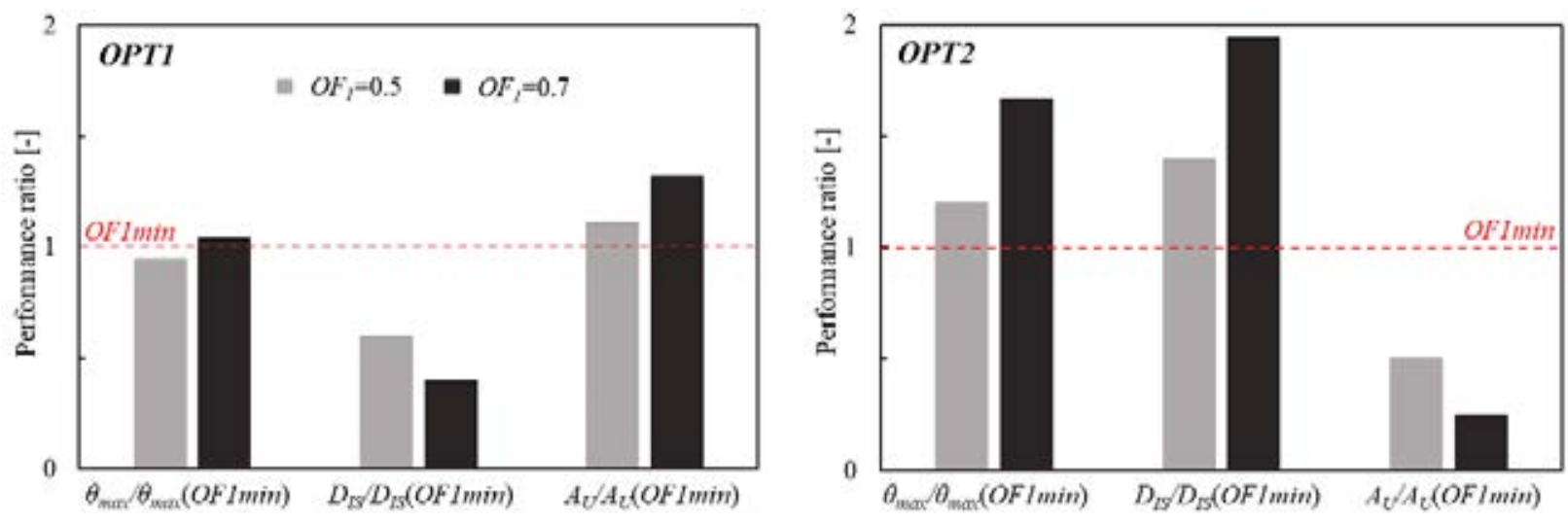

Figure 12: Average structural responses (for the cases $O F_{l}=0.5$ and 0.7 ) normalized to the case of $O F_{1 m i n}$.

\section{CONCLUSIONS}

A study investigating the inter-storey isolation system (IIS) as a technique for adding upper storeys to an existing building, while improving its seismic behaviour, is presented. A 3storey case study structure (without considering the IIS) was taken as a reference.

The first part of the study evaluated the effects of the main parameters of the IIS (or nonconventional TMD) on the dynamic response of the substructure; these parameters are the ratios of damping $\left(\xi_{I S}\right)$, frequency $(v)$ and mass $(\mu)$, with $v$ and $\mu$ referring to the substructure characteristics. Given the prevailing "mass damping" behaviour for $\mu$ values $\leq 1$, the structure was modelled with a reduced-order 2 DOF model. The evaluation was performed in the frequency domain, using the complex modal analysis and the frequency response functions 
(FRFs) of the substructure drift, the isolation drift and the superstructure acceleration. The variations of the characteristics of the second vibration mode and of the FRFs were evaluated over the entire range assumed for the various parameters of the IIS (i.e., $\xi_{I S}$ from 0 to 2, $v$ and $\mu$ from 0 to 1 ). These preliminary assessments led to the subsequent definition of the methodology to optimize the IIS parameters.

In particular, two alternative multi-objective optimizations were proposed, named OPT1 and $O P T 2$, minimizing different objective functions (OFs). OPT1 minimizes the variance of the substructure drift $\left(O F_{1}\right)$ along with that of the isolation drift $\left(O F_{2}\right)$; OPT2 still minimizes the variance of the substructure drift $\left(O F_{1}\right)$, but together with the minimization of the acceleration variance of the superstructure $\left(\mathrm{OF}_{3}\right)$.

The NSGA-II algorithm was used to solve the multi-objective problem, searching for the best solutions of $v$ and $\xi_{I S}$ for set $\mu$ values. The solutions obtained from OPT1 and OPT2 were compared with each other, as well as with other relevant solutions from the literature. To this end, representative optimal solutions were selected from the Pareto fronts on the basis of the value of $O F_{1}$, i.e. $O F_{1}=O F_{1 \min }(\approx 0.4), 0.5$ and 0.7 .

Finally, based on these optimal solutions, time-history analyses were performed, in order to verify the effectiveness of the optimization approach and therefore the beneficial effects of the IIS technique. For this purpose, a set of natural accelerograms was assumed, which is compatible with the EC8-Type1 spectrum, with soil B and PGA=0.3 $g$, and the substructure was modelled as a lumped mass 3 DOF system with a non-linear structural behaviour. Overall, the following conclusions can be drawn:

- The IIS technique, when its stiffness $(v)$ and damping $\left(\xi_{I S}\right)$ parameters are suitably optimized in relation to the isolated mass ratio $(\mu)$, seems effective in improving the seismic behaviour of the substructure (or existing structure).

- Considering the optimal solution that minimizes the variance of the substructure drift, i.e. $O F_{1 \min }$, the increase in the mass ratio (if allowed by the substructure in terms of vertical capacity) leads to a reduction of the optimal values of $v$ and to an increase of those of $\xi_{I S}$, i.e. solutions that are more similar to those of seismic isolation. Consequently, increasing $\mu$ reduces the accelerations of the superstructure but increases the drift of the isolation system.

- The OPT1 optimization provides effective solutions to reduce the isolation drift while containing the maximum values of the substructure drift similar to those of the $O F_{1 \text { min }}$ solution. With the solutions of $O P T 1$, a more uniform behaviour of the substructure is also obtained, minimizing floor accelerations.

- The OPT2 optimization provides effective solutions to reduce the superstructure acceleration while containing the maximum values of the substructure drift similar to those of the $O F_{1 \min }$ solution. With the solutions of $O P T 2$, a more decouple behaviour between the two structural parts is also obtained, and therefore a lower reduction in the substructure accelerations.

- $O P T 1$ is particularly advantageous for the higher $\mu$ values (for which the isolation drift is more sensitive $-O F_{2}$ ), whereas $O P T 2$ for the lower $\mu$ values (for which the superstructure acceleration is more sensitive $-O F_{3}$ ). Finally, the solutions for $O F_{1}=0.5$ seem a good compromise for both optimizations. 


\section{REFERENCES}

[1] R. Zhang, B.M. Phillips, S. Taniguchi, M. Ikenaga, K. Ikago, Shake table real-time hybrid simulation techniques for the performance evaluation of buildings with inter-story isolation. Structural Control Health Monitoring $24 . \quad 1013$ https://doi.org/10.1002/stc.1971, 2017

[2] Y. Liu, J. Wu, M. Donà, Effectiveness of fluid-viscous dampers for improved seismic performance of inter-storey isolated buildings. Engineering Structures 169:276-292. https://doi.org/10.1016/j.engstruct.2018.05.031, 2018

[3] F. L. Zhou, Seismic isolation of civil buildings in the People's Republic of China. Progress in Structural Engineering and Materials 1015 3(3):268-276. https://doi.org/10.1002/pse.85, 2001

[4] M. Chey, J. Geoffrey Chase, J. Mander, A. Carr, Innovative seismic retrofitting strategy of added stories isolation system. Frontiers of Structural and Civil Engineering, 7, 13 23. https://doi.org/10.1007/s11709-013-0195-9, 2013

[5] D. Faiella, B. Calderoni, E. Mele, Seismic Retrofit of Existing Masonry Buildings through Inter-story Isolation System: A Case Study and General Design Criteria. Journal of Earthquake Engineering, DOI: 10.1080/13632469.2020.1752854, 2020

[6] M. Ziyaeifar, T Noguchi, Partial mass isolation in tall buildings. Earthquake Engineering and Structural Dynamics, https ://doi.org/10.1002/(SICI)1096-9845(19980 1)27:1\%3c49:AID-EQE718\%3e3.0.CO;2-J, 1998

[7] P. Tan, Y. Zhang, F. Zhou, Optimal design and control mechanism study on story isolation system. The $14^{\text {th }}$ World Conference on Earthquake Engineering, Beijing, China, October 12-17, 2008

[8] S. J. Wang, K. C. Chang, J. S. Hwang, B. H. Lee, Simplified analysis of mid-story seismically isolated buildings. Earthquake Engineering and Structural Dynamics, https://doi.org/10.1002/eqe.1004, 2011

[9] S. J. Wang, J. S. Hwang, K. C. Chang, M. H. Lin, B. H. Lee, Analytical and experimental studies on midstory isolated buildings with modal coupling effect. Earthquake Engineering and Structural Dynamics, https://doi.org/10.1002/eqe.2203, 2013

[10] D.C. Charmpis, P. Komodromos, M.C. Phocas, Optimized earthquake response of multistorey buildings with seismic isolation at various elevations. Earthquake Engineering and Structural Dynamics, https://doi.org/10.1002/eqe.218, 2012

[11] D.C. Charmpis, M.C. Phocas, P. Komodromos, Optimized retrofit of multi-storey buildings using seismic isolation at various elevations: assessment for several earthquake excitations. Bulletin of Earthquake Engineering, https://doi.org/10.1007/s10518-0159737-y, 2015

[12] D. Faiella, E. Mele, Vibration characteristics and higher mode coupling in intermediate isolation systems (IIS): a parametric analysis. Bulletin of Earthquake Engineering DOI:10.1007/s10518-019-00637-w, 2019

[13] M. Donà, E. Bernardi, A. Zonta, M. Minotto, F. da Porto, P. Tan, Effectiveness and optimal design of fluid-viscous dampers for inter-storey isolated buildings. In Proc., XVIII ANIDIS Conf., Ascoli Piceno, Italy, 2019 
[14] M. Donà, E. Bernardi, A. Zonta, P. Tan, F. Zhou, Evaluation of optimal FVDs for interstorey isolation systems based on surrogate performance models. Bulletin of Earthquake Engineering, 2021

[15] H. Frahm, Devices for Damped Vibrations of Bodies, U.S. Patent No. 989958, Oct 30, 1909

[16] J.P. Den Hartog, Mechanical Vibrations. McGraw-Hill, 3rd edition, 1947

[17] G.B. Warburton, Optimum absorber parameters for various combinations of response and excitation parameters. Earthquake Engineering and Structural Dynamics, 10(3):381-401, 1982

[18] H. Tsai, G. Lin, Optimum tuned-mass dampers for minimizing steady-state response of support-excited and damped systems. Earthquake Engineering and Structural Dynamics, 22(11):957-973, 1993

[19] R. Villaverde, Reduction in seismic response with heavily-damped vibration absorbers. Earthquake Engineering and Structural Dynamics, https://doi.org/10.1002/eqe.4290130105, 1985

[20] R. Rana, T.T. Soong, Parametric study and simplified design of tuned mass dampers. Engineering Structures, https://doi.org/10.1016/S0141-0296(97)00078-3, 1998

[21] M. Yahyai, L. Zebarjad, M. Head, M. Shokouhian, Optimum Parameters for Large Mass Ratio TMDs Using Frequency Response Function, Journal of Earthquake Engineering, https://doi.org/10.1080/13632469.2019.1624228, 2019

[22] G. Bekdaş, S. M. Nigdeli, Metaheuristic based optimization of tuned mass dampers under earthquake excitation by considering soil-structure interaction. Soil Dynamics and Earthquake Engineering 92, 443-461. doi:10.1016/j.soildyn.2016.10.019, 2017

[23] F. Sadek, B. Mohraz, A.W. Taylor, R.M. Chung, A method of estimating the parameters of tuned mass dampers for seismic applications. Earthquake Engineering and Structural Dynamics, 26(6):617-635, 1997

[24] J. Miranda, On tuned mass dampers for reducing the seismic response of structures. Earthquake Engineering and Structural Dynamics, 34:847-865. DOI: 10.1002/eqe.461, 2005

[25] C. Moutinho, An alternative methodology for designing tuned mass dampers to reduce seismic vibrations in building structures. Earthquake Engineering and Structural Dynamics, https://doi.org/10.1002/eqe.2174, 2012

[26] A. Reggio, M. De Angelis, Optimal energy-based seismic design of non-conventional Tuned Mass Damper (TMD) implemented via inter-story isolation. Earthquake Engineering and Structural Dynamics, https://doi.org/10.1002/eqe.2548, 2015

[27] D. Pietrosanti, M. De Angelis, M. Basili, Optimal design and performance evaluation of systems with Tuned Mass Damper Inerter (TMDI). Earthquake Engineering and Structural Dynamics, https://doi.org/10.1002/eqe.2861, 2017

[28] Q. Zhou, M.P. Singh, X.Y. Huang, Model reduction and optimal parameters of midstory isolation systems. Engineering Structures, https://doi.org/10.1016/j.engstruct.2016.06.011, 2016 
[29] M. Donà, A.H. Muhr, G. Tecchio, F. da Porto, Experimental characterization, design and modelling of the RBRL seismic-isolation system for lightweight structures. Earthquake Engineering and Structural Dynamics 46:831-853. 942 https://doi.org/10.1002/eqe.2833, 2017

[30] A. S. Veletsos, C. E. Ventura, Modal analysis of non-classically damped linear systems. Earthquake Engineering and Structural Dynamics, doi: 10.1002/eqe.4290140205, 1986

[31] K. Deb, A. Pratap, S. Agarwal, T. Meyarivan, A fast and elitist multiobjective genetic algorithm: NSGA-II. IEEE Transactions on Evolutionary Computation, https://doi.org/10.1109/4235.996017, 2002

[32] C. Coello Coello, G.B. Lamont, D. Van Veldhuizen, Evolutionary Algorithms for Solving Multi-Objective Problems. Genetic and Evolutionary Computation Series, 2007

[33] N. Ambraseys, P. Smit, R. Sigbjornsson, P. Suhadolc, B. Margaris, Internet-Site for European Strong-Motion Data. European Commission, Research-Directorate General, Environment and Climate Programme, 2002 